\title{
Singularities of projected immersions revisited
}

\author{
GÁBOR LIPPNER
}

\begin{abstract}
Szúcs proved in [6] that the $r$-tuple-point manifold of a generic immersion is cobordant to the $\Sigma^{1 r-1}$-point manifold of its generic projection. Here we extend this by showing that the natural mappings of these manifolds are bordant to each other. The main novelty of our approach is that we construct an explicit geometric realization of the bordism.
\end{abstract}

$57 \mathrm{R} 42,57 \mathrm{R} 45$

\section{Introduction}

There is a surprising relationship between the multiple-points of an immersion $g: M \hookrightarrow \rightarrow$ $N \times \mathbb{R}$ and the singularities of its projection $f: M \rightarrow N$ that was found by Szúcs [6] (see also Szúcs [5]). Namely he showed that if $N$ is a Euclidean space then the $(r+1)$-tuple-points of $g$ are cobordant to the $\Sigma^{1_{r}}$ points of $f$. The case $r=2$ has long been well-known (see eg McCrory [3]), and the case of higher singularities was also studied by McCrory [2].

The proof of this result involved computing the characteristic numbers of the two manifolds and observing that they coincide. It is very natural to ask whether this cobordism can be "seen" in an explicit way hidden in the geometry of $f$, not just as mere luck that all the characteristic numbers coincide.

We shall answer this question in the affirmative by constructing a cobordism that connects the two manifolds. This allows us to slightly extend the original theorem: instead of cobordism of manifolds we obtain singular bordism of maps, and we prove the theorem for any smooth target manifold $N$. (The notation and the necessary definitions are given in Section 2.)

Theorem 1.1 Let $f: M^{n} \rightarrow N^{n+k}$ be a prim map, and let $g: M \leftrightarrow \rightarrow \times \mathbb{R}$ be its lift to an immersion. Then for any $r \geq 1$ we have $g_{r} \sim\left(\Sigma^{1_{r-1}}(f) \hookrightarrow M\right)$, that is they represent the same element in the singular bordism group $\mathcal{N}_{*}(M)$.

If $M$ and $N$ are oriented and the codimension $k$ is odd, then $g_{r} \sim_{\mathrm{SO}}\left(\Sigma^{1_{r-1}}(f) \hookrightarrow M\right)$, that is they represent the same element in the singular oriented bordism group $\Omega(M)$. 
This research was partially supported by grant T049449 of the Hungarian Scientific Research Fund.

\section{Definitions and notation}

Consider a proper, generic immersion $g: M^{n} \rightarrow N^{n+k}$ of a closed smooth manifold $M$ to a smooth manifold $N$. The $r$-fold points of $g$ are those points in $N$ whose preimage consists exactly of $r$ different points. We shall denote this set $N_{r}$. This is not always a closed set in $N$. Its closure $\bar{N}_{r}$ consists of those points that have at least $r$ distinct preimages. Put $M_{r}=g^{-1}\left(N_{r}\right)$, this is the set of $r$-tuple points of $g$ in the source manifold. Its closure is denoted by $\bar{M}_{r}$.

The sets $M_{r}$ and $N_{r}$ are generally not submanifolds of $M$ and $N$ but they are images of (nongeneric) immersions of (possibly open) manifolds. Here we recall a well-known construction (see eg Ronga [4]) to fix the notation: Let

$$
\widehat{M}_{r}(g)=\left\{\left(x_{1}, \ldots, x_{r}\right) \in M^{(r)}: g\left(x_{1}\right)=\cdots=g\left(x_{r}\right),(i \neq j) \Rightarrow\left(x_{i} \neq x_{j}\right)\right\} .
$$

The symmetric group $S_{r}$ acts on this set freely in an obvious way. Let $\left[x_{1}, \ldots, x_{r}\right]$ denote the equivalence class of $\left(x_{1}, \ldots, x_{r}\right)$. On the other hand $S_{r-1}$ also acts freely on the last $r-1$ coordinates. Here the equivalence class of $\left(x_{1}, \ldots, x_{r}\right)$ is denoted by $\left(x_{1},\left[x_{2}, \ldots, x_{r}\right]\right)$.

Definition 2.1 The sets of equivalence classes are denoted by

$$
\begin{aligned}
& \widetilde{\Delta_{r}}(g)=\widehat{M}_{r}(g) / S_{r} \\
& \Delta_{r}(g)=\widehat{M}_{r}(g) / S_{r-1} .
\end{aligned}
$$

There are obvious mappings

$$
\begin{array}{lrl}
\tilde{g_{r}}: \widetilde{\Delta_{r}}(g) \rightarrow N & \tilde{g_{r}}\left(\left[x_{1}, \ldots, x_{r}\right]\right):=g\left(x_{1}\right) \\
g_{r}: \Delta_{r}(g) \rightarrow M & g_{r}\left(x_{1},\left[x_{2}, \ldots, x_{r}\right]\right):=x_{1} \\
s_{r}: \Delta_{r}(g) \rightarrow \widetilde{\Delta_{r}}(g) & s_{r}\left(x_{1},\left[x_{2}, \ldots, x_{r}\right]\right):=\left[x_{1}, \ldots, x_{r}\right] .
\end{array}
$$

The images of $\widetilde{g_{r}}$ and $g_{r}$ are clearly $\bar{N}_{r}$ and $\bar{M}_{r}$ and they are bijective to the points that have multiplicity exactly $r$. On the other hand $s_{r}$ is clearly an $r$-sheeted covering.

The sets $\widetilde{\Delta_{r}}(g)$ and $\Delta_{r}(g)$ are called the $r$-fold multiple-point manifolds of $g$ in the target and source respectively. They are indeed manifolds. To see this we need the notion of the fat and narrow diagonals. Let $V$ be a manifold and let $V^{(r)}$ be its $r$-fold Cartesian product. Then let $\delta_{r}(V)=\left\{(x, x, \ldots, x) \in V^{(r)} \mid x \in V\right\}$ and 
$\Delta_{r}(V)=\left\{\left(x_{1}, x_{2}, \ldots, x_{r}\right) \in V^{(r)} \mid \exists i \neq j, x_{i}=x_{j}\right\}$ denote the narrow and the fat diagonals respectively. Consider the $r$-fold product $g^{(r)}: M^{(r)} \rightarrow N^{(r)}$. Clearly

$$
\widehat{M}_{r}(g)=\left(g^{(r)}\right)^{-1}\left(\delta_{r}(N)\right) \backslash \Delta_{r}(M) .
$$

Since $g$ is a generic immersion, $g^{(r)}$ is transverse to $\delta_{r}(N)$ and thus $\widehat{M}_{r}(g)$ is a closed manifold of dimension $n-(r-1) k$. The symmetric group $S_{r}$ acts on it freely, so after factoring out with the group actions of $S_{r}$ or $S_{r-1}$ we still get manifolds.

Remark If $M$ and $N$ are oriented and the codimension is even, then the multiplepoint manifolds can be given a natural orientation. If $k$ is odd however, then the action of $S_{r}$ contains orientation reversing elements, thus the factor manifolds will have no, or at least no preferred orientation.

Definition 2.2 Given a smooth map $f: M \rightarrow N$ where $\operatorname{dim} M \leq \operatorname{dim} N$, a point $x \in M$ is said to be a $\Sigma^{i}$ point if the corank (ie the dimension of the kernel) of $d f_{x}: T_{x} M \rightarrow T_{f(x)} N$ is $i$. The closure of the set of such points will be denoted by $\Sigma^{i}(f)$. If $i_{1} \geq i_{2}$ then we can define $\Sigma^{i_{1}, i_{2}}(f)=\Sigma^{i_{2}}\left(f \mid \Sigma^{i_{1}}(f)\right)$. This method can be continued recursively to give the definition of $\Sigma^{\left(i_{1}, i_{2}, \ldots, i_{r}\right)}$ points, where $i_{1} \geq i_{2} \geq$ $\cdots \geq i_{r}$. This classification of singular points is called the Thom-Boardman type. For details see eg Arnold et al [1].

Definition 2.3 A map $f: M \rightarrow N$ is said to be a Morin map if it has no $\Sigma^{2}$ points. The singularities of such maps are classified by their Thom-Boardman type, which can only be

$$
\Sigma \overbrace{(1,1, \ldots, 1)}^{r}=\Sigma^{1 r}
$$

for some $r \geq 0$. (In the notation of [1] this is $A_{r}$.) The set $\Sigma^{1^{1}}(f)$ is actually a submanifold of $M$.

Definition 2.4 A generic map $f: M \rightarrow N$ is called prim (projected immersion) if it has a specified lifting to a generic immersion, $g: M \leftrightarrow \rightarrow \times \mathbb{R}$ (ie $f=\pi \circ g$, where $\pi: \times \mathbb{R} \rightarrow N$ is the projection). This lifting $g$ has to be given up to regular homotopy. Such a map is necessarily a Morin map (i.e. its differential has corank at most one at any point), and so its singularities are classified by their Thom-Boardman type.

Remark Given a Morin map $f: M \rightarrow N$ one can associate to it its kernel-bundle, that is a line bundle over the singular points in $M$. It is not too hard to see that $f$ has a lifting to an immersion $g: M \leftrightarrow N \times \mathbb{R}$ if and only if this kernel-bundle is trivial (see eg Szúcs [5]). However the kernel-bundle can be trivialized in more than one way. 
Each trivialization gives a lifting to an immersion and every lifting gives a trivialization. It turns out that fixing the trivialization corresponds to fixing the regular homotopy class of the lifting. Generally it is more convenient to assume that the kernel-bundle has a fixed trivialization rather than just being trivial, hence the usual definition of prim map is a Morin map with trivializes kernel-bundle. In the lifting terminology this means that two liftings that are not regularly homotopic in the space of all possible liftings represent different prim maps. Though in our context both approaches work equally since we shall work with a completely fixed lifting.

Definition 2.5 The fat diagonal of $\Sigma^{1^{1}}(f) \times M^{(i-1)}$ can be defined analogously to $\Delta_{i}(M)$, since $\Sigma^{1^{1}}(f) \subset M$ is a submanifold. Let us denote

$$
\Delta_{i}^{r}(M)=\left\{\left(x_{1}, x_{2}, \ldots, x_{i}\right) \in \Sigma^{1 r}(f) \times M^{(i-1)}: \exists j \neq l, x_{j}=x_{l}\right\} .
$$

Remark For any manifold $M$ we shall denote its cobordism class by $[M] \in \mathcal{N}_{*}$ and for a map $f: M \rightarrow N$ we shall denote its singular bordism class by $[f] \in \mathcal{N}_{*}(N)$. The cobordism relation for both manifolds and maps will be denoted by $\sim$. If $M$ is oriented then the same notation will be used for the corresponding classes in $\Omega_{*}$ and $\Omega_{*}(N)$ respectively.

\section{Proof of Theorem 1.1}

\subsection{Preparations}

Let us fix a prim map $f: M^{n} \rightarrow N^{n+k}$, its lift $g: M \hookrightarrow \rightarrow N \times \mathbb{R}$ and an integer $r \geq 2$ (for $r=1$ the statement is obvious). For any $1 \leq i \leq r$ we shall introduce an auxiliary manifold $\Lambda_{r}^{i}$ and a map $\lambda_{r}^{i}: \Lambda_{r}^{i} \rightarrow M$ which we shall call the "mixed"-point manifold. Mixed in the sense that these $\lambda_{r}^{i}$ will be resolutions of those points in $M$ that are $i$-tuple points of $g$ and at the same time $\Sigma^{1_{r-i}}$ points of $f$. These points do not necessarily form a submanifold of $M$, but we can construct their resolution just like we did for the set of $r$-tuple points of an immersion: Let us consider the map $G_{i}:=\left.g\right|_{\Sigma^{1}-i}(f) \times g \times \cdots \times g: \Sigma^{1_{r-i}}(f) \times M \times \cdots \times M \rightarrow(N \times \mathbb{R}) \times \cdots \times(N \times \mathbb{R})$, where we take $i-1$ factors of $M$ on the left, and thus $i$ factors of $(N \times \mathbb{R})$ on the right. Since $f$ is a generic prim map and $g$ is its generic lift we have that outside of the fat diagonal (see Definition 2.5) $\Delta_{i}^{r-i}(M) \subset \Sigma^{1_{r-i}}(f) \times M^{(i-1)}$ the map $G_{i}$ is transverse to the narrow diagonal $\delta_{i}(N \times \mathbb{R}) \subset(N \times \mathbb{R})^{(i)}$. Let us consider the set $\widehat{M}_{i, r-i}(f):=G_{i}^{-1}\left(\delta_{i}(N \times \mathbb{R})\right) \backslash \Delta_{i}^{r-i}(M)$. The transversality of $G_{i}$ implies that this 
set is a manifold without boundary. But $g$ is an immersion and $G_{i}$ is obtained by restricting $g$ to a certain submanifold. From this it follows by Lemma 2.1 on page 523 of [4] (or as a special case of Theorem 4.2 in Section 4 of this paper) that $\widehat{M}_{i, r-i}(f)$ is actually disjoint from a small open neighborhood of the fat diagonal $\Delta_{i}^{r-i}(M)$. Hence it is compact and thus it is actually a closed submanifold of $\Sigma^{1^{r-i}}(f) \times M^{(i-1)}$. The symmetric group $S_{i-1}$ acts on $\Sigma^{1_{r-i}}(f) \times M^{(i-1)}$ by permuting the last $i-1$ coordinates. This action restricted to $\widehat{M}_{i, r-i}(f)$ is free, so we can factorize and get the manifold

$$
\Lambda_{r}^{i}=\widehat{M}_{i, r-i}(f) / S_{i-1} .
$$

A point of $\Lambda_{r}^{i}$ can be referred to as $\left(x_{1},\left[x_{2}, \ldots, x_{i}\right]\right)$ where the $x_{j}$ 's are all different, $g\left(x_{1}\right)=g\left(x_{2}\right)=\cdots=g\left(x_{i}\right)$ and $x_{1} \in \Sigma^{1^{1-i}}(f)$. In this notation the desired resolution

$$
\lambda_{r}^{i}: \Lambda_{r}^{i} \rightarrow M
$$

is given by

$$
\left(x_{1},\left[x_{2}, \ldots, x_{i}\right]\right) \mapsto x_{1} .
$$

(The maps $f, g$ are omitted from the notation.) It is easy to see that the manifold $\Lambda_{r}^{i}$ has dimension $n-(r-1)(k+1)$. If we choose $i=r$ then we get that $G_{i}$ is the product map $g^{(r)}$ and hence our construction boils down to the standard original construction of the $r$-tuple point manifold. So $\Lambda_{r}^{r}=\Delta_{r}(g)$ and $\lambda_{r}^{r}=g_{r}$. In the other extreme case, when $i=1$ we get that $G_{i}$ is simply the inclusion $\Sigma^{1^{r-1}}(f) \hookrightarrow N \times \mathbb{R}$. The narrow diagonal of the right hand side is the whole space and the fat diagonal of the left hand side is empty. Hence the mixed-point manifold becomes just the singular set and its "resolution" $\lambda_{r}^{1}: \Lambda_{r}^{1} \rightarrow M$ is the natural inclusion $\Sigma^{1_{r-1}}(f) \hookrightarrow M$. Thus the theorem follows from the following lemma.

Lemma 3.1 $\lambda_{r}^{1} \sim \lambda_{r}^{2} \sim \cdots \sim \lambda_{r}^{r}$, ie these maps represent the same class in $\mathcal{N}_{*}(N)$.

The proof consists of two very different ingredients. The first ingredient is the global construction of the desired cobordisms using the map $f$. The constructed spaces are easy to describe but they are not obviously manifolds. The precise proof that they are indeed manifolds requires detailed study of the map $f$ near its singular points. Thus the second ingredient is a local computation using normal forms. This computation is only a technical point so first we give the proofs omitting the computational details. Then in Section 4 we finally show how to carry out the computations used earlier.

\subsection{A sample case}

Here we shall illustrate the main idea behind Lemma 3.1 in a special case where it is actually possible to visualize both the immersion and its projection. The only case that 
is interesting and can be "drawn" at the same time is that of an immersion of a surface into $\mathbb{R}^{3}$ and its projection to $\mathbb{R}^{2}$.

Instead of using a closed surface as an example, we shall just use a small setup which should be thought of as being a part of a large example. In the following pictures we sketch the immersion of an open disc to $\mathbb{R}^{3}$. The projection should be understood to be the vertical one. We start by taking the picture of a usual cusp lifted to $\mathbb{R}^{3}$ as in Figure 1.

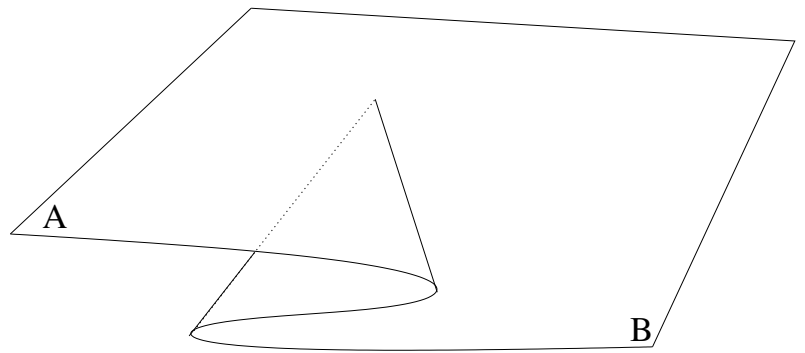

Figure 1: A usual cusp

Then we take the corner labeled "A", and pull it down so as to create self-intersection. We then do the same with the corner "B", only upwards. The resulting immersion is shown in Figure 2.

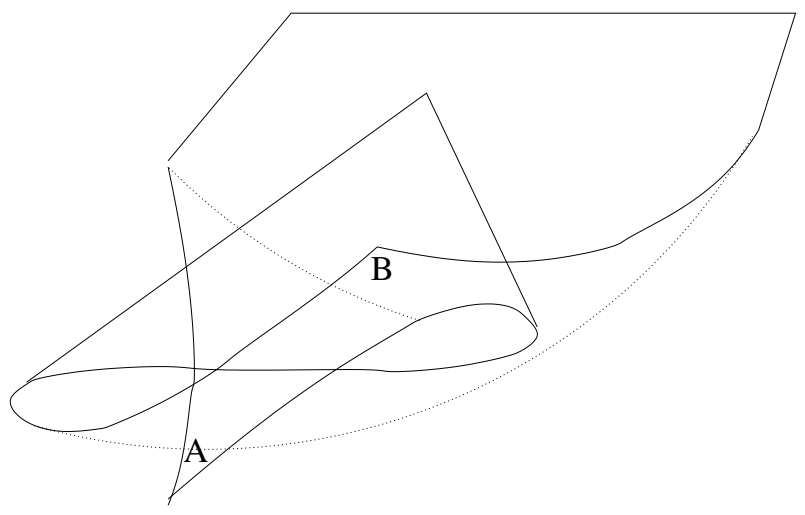

Figure 2: Moving the corners up and down

On Figure 3 we can see the same immersion with two dashed lines representing the self-intersection. Where these two dashed lines meet there is a triple point ("T"). The third dashed line labeled with "e" is the vertical projection, or shadow of one of the 


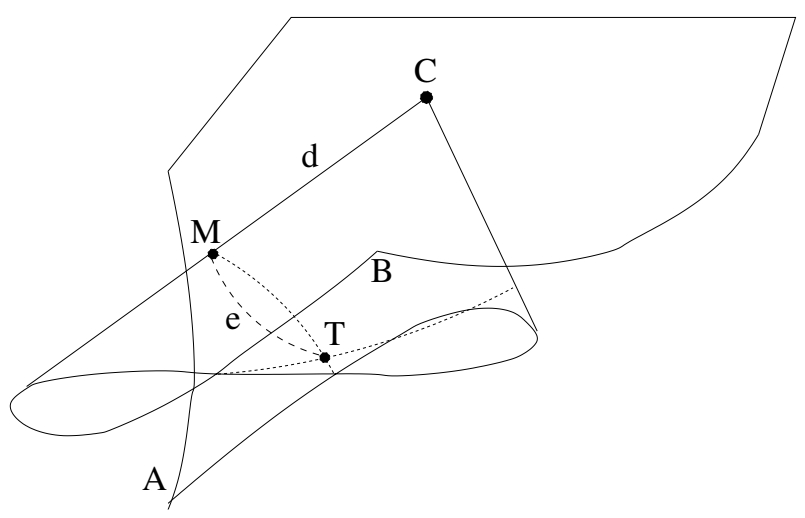

Figure 3: Dashed lines indicate self-intersection points

self-intersection lines to the layer of corner "B". This line is the set of those points above which there is a double-point of the immersion.

The cusp point of the projection is labeled "C" on the picture. "M" shows the place of one of the mixed-points. Namely it is a double-point and a fold point at the same time. The segment "e" provides the cobordism between "M" and "T". It is a manifold with two types of boundary. This follows from transversality and the local computations in Section 4. The first type, when the double-point curve intersects the sheet on which "e" lies creates a boundary of "e" and that exactly corresponds to a triple-point. The other type is when one of the sheets forming the double-curve comes together with the sheet containing "e". This also creates a boundary, which will be a mixed-point. This way "e" forms the cobordism between the triple-points and the mixed-points.

Finally the arc labeled by "d" consist of points that are fold points above which there is an other sheet of the immersion. As before, by transversality and the local computations it turns out that this is always a manifold with two types of boundary. The first type occurs when the sheet above the fold curve passes through the fold curve thereby creating a mixed-point. The other type occurs when the sheet above the fold curve comes together with the fold curve, creating a cusp point. Thus "d" provides the cobordism between the cusps and the mixed-points.

\subsection{Proof of Lemma 3.1}

We shall now turn to the precise construction of the cobordism manifolds in the general case. Let us again consider the map

$G_{i}:=\left.g\right|_{\Sigma^{1}-i}(f) \times g \times \cdots \times g: \Sigma^{1_{r-i}}(f) \times M \times \cdots \times M \rightarrow(N \times \mathbb{R}) \times \cdots \times(N \times \mathbb{R})$. 
Let us define

$$
\Delta_{i}^{+}=\left\{((x, s),(x, t), \ldots,(x, t)) \in(N \times \mathbb{R})^{(i)}: s \geq t\right\} .
$$

Outside of $\Delta_{i}^{r-i}(M)$ the map $G_{i}$ is transverse to $\Delta_{i}^{+}$and $\partial \Delta_{i}^{+}=\delta_{i}(N \times \mathbb{R})$, since both $f$ and $g$ are generic and thus self-transverse.

Let us now define $H^{\prime}=G_{i}^{-1}\left(\Delta_{i}^{+}\right) \backslash \Delta_{i}^{r-i}(M)$. Transversality implies that $H^{\prime}$ is a (not necessarily compact) manifold with boundary $G_{i}^{-1}\left(\delta_{i}(N \times \mathbb{R})\right) \backslash \Delta_{i}^{r-i}(M)=$ $\widehat{M}_{i, r-i}(f)$. Let us denote the closure of $H^{\prime}$ in $\Sigma^{1_{r-i}}(f) \times M^{(i-1)}$ by $H$. Obviously $H \backslash H^{\prime} \subset \Delta_{i}^{r-i}(M)$. We have seen in Section 3.1 that $\partial H^{\prime}$ is a closed manifold disjoint from the fat diagonal. Thus $\partial H^{\prime}$ is disjoint from $H \backslash H^{\prime} \subset \Delta_{i}^{r-i}(M)$.

Let us take a point $\left(x_{1}, \ldots, x_{i}\right) \in H \backslash H^{\prime}$. Then by definition of $H^{\prime}$ there exist points $y_{j}^{k}(k \geq 1, i \geq j \leq 1)$ that fulfill all the following requirements:

(1) For every $j$ we have $\lim _{k \rightarrow \infty} y_{j}^{k}=x_{j}$.

(2) $y_{1}^{k} \in \Sigma^{1_{r-i}}(f)$.

(3) For any fixed $k$ the $y_{j}^{k}$,s are all different.

(4) $g\left(y_{j_{1}}^{k}\right)=g\left(y_{j_{2}}^{k}\right)$ for any $j_{1}, j_{2} \geq 2$.

(5) $f\left(y_{j_{1}}^{k}\right)=f\left(y_{j_{2}}^{k}\right)$ for any $j_{1}, j_{2} \geq 1$.

Since $g$ is a generic immersion, (3) and (4) imply that for all $j>l \geq 2$ we have $x_{j} \neq x_{l}$. Then since $H \backslash H^{\prime} \subset \Delta_{i}^{r-i}(M)$ there must be a $j>1$ such that $x_{1}=x_{j}$. Thus $y_{1}^{k} \rightarrow x_{1}$ and $y_{j}^{k} \rightarrow x_{1}$ as well. Furthermore $y_{1}^{k} \in \Sigma^{1^{1-i}}(f)$. Theorem 4.2 in Section 4 can be applied and hence $x_{1} \in \Sigma^{1_{r-i+1}}(f)$.

Conversely let us suppose that $x_{1} \in \Sigma^{1^{r-i+1}}(f)$ and $x_{2}, \ldots, x_{i-1}$ are all different from each other and $x_{1}$ and $g\left(x_{j}\right)$ is the same for every $1 \leq j \leq i-1$. We want to show that in the neighborhood of $\left(x_{1}, x_{1}, x_{2}, \ldots, x_{i-1}\right)$ the set $H$ is a compact manifold with boundary and $\left(x_{1}, x_{1}, x_{2}, \ldots, x_{i-1}\right)$ is on $\partial H$. First consider the first two factors separately from the others.

$$
G_{2}=\left.g\right|_{\Sigma^{1_{r-i}}(f)} \times g: \Sigma^{1_{r-i}}(f) \times M \rightarrow(N \times \mathbb{R})^{(2)} .
$$

Let us denote $H_{2}^{\prime}=G_{2}^{-1}\left(\Delta_{2}^{+}\right)$. By Theorem 4.3 in Section 4 we know that locally around $\left(x_{1}, x_{1}\right)$ its closure $H_{2}=\operatorname{cl}\left(H_{2}^{\prime}\right)$ is a compact manifold with boundary $\partial H_{2}=$ $\left\{(u, u): u \in \Sigma^{1_{r-i+1}}(f)\right\}$. Clearly $H$ is locally the complete intersection of $H_{2} \subset$ $\Sigma^{1_{r-i}}(f) \times M$ around $\left(x_{1}, x_{1}\right)$ and $\widehat{M}_{i-2}(g) \subset M^{(i-2)}$ around $\left(x_{2}, \ldots, x_{i-1}\right)$. Thus the genericity of $f$ and $g$ implies that $H$ is also locally a compact manifold with boundary $\partial H$ the complete intersection of $\partial H_{2}$ and $\widehat{M}_{i-2}(g)$. 
Thus $H$ is a compact manifold. Its boundary consist of two disjoint components $H \backslash H^{\prime}$ and $\partial H^{\prime}=\widehat{M}_{i, r-i}(f)$. The symmetric group $S_{i-1}$ acts on $\Sigma^{1_{r-i}}(f) \times M^{(i-1)}$ by permuting the last $i-1$ coordinates. By definition $H^{\prime}$ is invariant under this action. The above considerations show that $\partial H^{\prime}$ and $H \backslash H^{\prime}$ are also invariant, and the action is free on each. Thus we can factorize by this action on $H$ and get that the quotient is again a compact manifold $\hat{H}$ with boundary $\partial H^{\prime} / S_{i-1}$ and $\left(H \backslash H^{\prime}\right) / S_{i-1}$. By definition $\partial H^{\prime} / S_{i-1}=\widehat{M}_{i, r-i}(f) / S_{i-1}=\Lambda_{r}^{i}$. On the other hand we have seen that

$$
\begin{array}{r}
H \backslash H^{\prime}=\left\{\left(x_{1}, x_{2}, \ldots, x_{i}\right) \in \Delta_{i}^{r-i+1}(M) \backslash \Sigma^{1_{r-i+1}}(f) \times \Delta_{i-1}(M):\right. \\
\left.g\left(x_{j}\right)=g\left(x_{l}\right)(1 \leq j<l \leq i)\right\} .
\end{array}
$$

So there is a natural map $\phi:\left(H \backslash H^{\prime}\right) / S_{i-1} \rightarrow \Lambda_{r}^{i+1}$ given by $\phi\left(x_{1},\left[x_{2}, \ldots, x_{i}\right]\right)=$ $\left(x_{1},\left[x_{2}, \ldots, x_{j-1}, x_{j+1}, \ldots, x_{i}\right]\right)$ when $x_{1}=x_{j}$. This map is clearly a diffeomorphism. Thus $\left(H \backslash H^{\prime}\right) / S_{i-1}=\Lambda_{r}^{i+1}$.

Finally projecting everything to the first coordinate we get a map $\hat{H} \rightarrow M$ that on the boundary coincides with $\lambda_{r}^{i}$ and $\lambda_{r}^{i+1}$. Thus $\lambda_{r}^{i} \sim \lambda_{r}^{i+1}$.

Remark If the codimension $k$ is odd, then the codimension of $g$ is even. So if $M$ and $N$ are oriented, then $H^{\prime}$ can be given a natural orientation. This is preserved by the action of $S_{i-1}$ and so the manifold $\hat{H}$ that creates the cobordism between $\lambda_{r}^{i}$ and $\lambda_{r}^{i+1}$ is oriented. Thus $\lambda_{r}^{i} \sim_{\mathrm{SO}} \lambda_{r}^{i+1}$ and the oriented part of the theorem follows as well.

\section{Local computations}

Let us consider a prim map $f: M^{n} \rightarrow N^{n+k}$. Let us write $n=r(k+1)+z$. Then the $\Sigma^{1 r}$-points of $f$ form a $z$-dimensional submanifold in $M$. Let $x \in$ $\Sigma^{1_{r}}(f) \backslash \Sigma^{1_{r+1}}(f)$. Then (according to eg [1]) it is possible to take small Euclidean neighborhoods of $x$ and $f(x)$ and introduce local coordinates such that $f$ takes the following local normal form (we take both $x$ and $f(x)$ to be in the origin):

$$
\begin{aligned}
F:\left(\mathbb{R}^{r(k+1)+z}, 0\right) & \rightarrow\left(\mathbb{R}^{1+k+(r(k+1)-1)+z}, 0\right) \\
\left(t, \bar{y}_{r}, \bar{y}_{r-1}, \ldots, \bar{y}_{1}, \bar{s}\right) & \mapsto\left(p_{0}(t), p_{1}(t), \ldots, p_{k}(t), \overline{\bar{y}}, \bar{s}\right),
\end{aligned}
$$

where

$$
\begin{aligned}
& \bar{y}_{j}=\left(y_{j, 0}, y_{j, 1}, \ldots, y_{j, k}\right) \in \mathbb{R}^{k+1} \\
& \bar{y}_{r}=\left(y_{r, 1}, y_{r, 2}, \ldots, y_{r, k}\right) \in \mathbb{R}^{k}
\end{aligned}
$$$$
\text { for every } 1 \leq j \leq r-1 \text {, }
$$ 
By $\overline{\bar{y}}$ we denote the collection of all $y_{i}^{j}$, so $\overline{\bar{y}} \in \mathbb{R}^{r(k+1)-1}$. Finally $\bar{s}=\left(s_{1}, \ldots, s_{z}\right) \in$ $\mathbb{R}^{z}$. The polynomials $p_{i}$ are defined by $p_{0}(t)=t^{r+1}+y_{r-1,0} t^{r-1}+\cdots+y_{1,0} t$ which is of degree $r+1$ and for any $i>0$ we have $p_{i}(t)=y_{r, i} t^{r}+\cdots+y_{1, i} t$ which is of degree $r$. We will think of the $p_{i}$ mostly as polynomials of the single variable $t$.

\section{Lemma 4.1}

(1) The point $(t, \overline{\bar{y}}, \bar{s})$ is a $\Sigma^{1_{j}}$-point of $F$ if and only if $p_{i}^{\prime}(t)=p_{i}^{\prime \prime}(t)=\cdots=$ $p_{i}^{(j)}(t)=0$ for every $0 \leq i \leq k$.

(2) The set of such points form a submanifold in $\mathbb{R}^{r(k+1)+z}$ which can be smoothly parametrized by $\bar{s}, \bar{y}_{r}, \ldots, \bar{y}_{j+1}$.

Proof Part (2) easily follows from part (1), since if $j<r$ and $\bar{s}, \bar{y}_{r}, \ldots, \bar{y}_{j+1}$ are fixed, then $p_{i}^{(j)}(t)=0$ is a nondegenerate linear equation for $\bar{y}_{j}$. This can be uniquely solved. Then $p_{i}^{(j-1)}(t)=0$ is a nondegenerate linear equation for $\bar{y}_{j-1}$, and so on. Finally if $j=r$ then obviously the only solution is $y_{l, i}=0$ for every $i, l$ independently of $\bar{s}$. Thus it suffices to show part (1).

We will proceed by induction on $j$. The initial step $j=1$ is easy to see: $d F$ is singular if and only if $p_{i}^{\prime}(t)=0$ for every $i$ and in this case ker $d F$ is the $t$-axis. Now let us suppose we know the statement for $j-1$ and take a point $x \in \Sigma^{1_{j}}(F)$. Then $x \in \Sigma^{1_{j-1}}(F)$ and $\operatorname{ker} d_{x} F \subset T_{x} \Sigma^{1_{j-1}}(F)$. Then there is a sequence of points $x(i)=\left(t(i), \overline{\bar{y}}(i), \bar{s}(i) \in \Sigma^{1_{j-1}}(F)\right.$ such that $x(i) \rightarrow x=(t, \overline{\bar{y}}, \bar{s})$,

and

$$
\begin{aligned}
& \frac{t(i)-t}{|x(i)-x|} \rightarrow 1 \\
& \frac{|\overline{\bar{y}}(i)-\overline{\bar{y}}|}{|x(i)-x|} \rightarrow 0 .
\end{aligned}
$$

Let us focus on $p_{l}$ where $l$ is arbitrary but fixed, and temporarily denote it by $p$. We will also temporarily include in the notation of $p$ all its hidden variables. Then

$$
\begin{aligned}
p^{(j)}(t, \overline{\bar{y}})=\lim _{i \rightarrow \infty} \frac{p^{(j-1)}(t(i), \overline{\bar{y}})-p^{(j-1)}(t, \overline{\bar{y}})}{t(i)-t} & \stackrel{(1)}{=} \lim _{i \rightarrow \infty} \frac{p^{(j-1)}(t(i), \overline{\bar{y}})-p^{(j-1)}(t(i), \overline{\bar{y}}(i))}{t(i)-t} \stackrel{(2)}{=} 0 .
\end{aligned}
$$

Here (1) holds since $p^{(j-1)}(t, \overline{\bar{y}})=p^{(j-1)}(t(i), \overline{\bar{y}}(i))=0$ by the inductive hypothesis. (2) holds since $p^{(j-1)}$ is a fixed finite sum of expressions linear in $\overline{\bar{y}}$ and

$$
\frac{|\overline{\bar{y}}(i)-\overline{\bar{y}}(i)|}{t(i)-t} \rightarrow 0 \text {. }
$$


This argument can be easily reversed and so the statement is true for $j$. This completes the induction and thus proves part (1).

Theorem 4.2 Let $f: M^{n} \rightarrow N^{n+k}$ a generic Morin map. If there exist points $x_{i} \neq x_{i}^{\prime} \in M ;(i \geq 1)$ such that $x_{i} \rightarrow x, x_{i}^{\prime} \rightarrow x, x_{i} \in \Sigma^{1_{r}}(f)$ and $f\left(x_{i}\right)=f\left(x_{i}^{\prime}\right)$ for every $i$, then $x \in \Sigma^{1 r+1}(f)$.

Proof It is obvious that $x \in \Sigma^{1_{r}}(f)$. Let us suppose that $x \in \Sigma^{1_{r}}(f) \backslash \Sigma^{1_{r+1}}(f)$. We can consider $f$ locally around $x$ and introduce Euclidean neighborhoods as before, denoting the function in the new coordinate-system by $F$. As $x_{i} \rightarrow x$ and $x_{i}^{\prime} \rightarrow x$, these points will fall into the chosen neighborhood with at most finite exceptions. From Lemma 4.1 it is obvious that the only $\Sigma^{1^{1}}$-points of $F$ are those for which $t=0$ and $\overline{\bar{y}}=\overline{\overline{0}}$, and $\bar{s}$ is arbitrary. On the other hand if $F(t, \overline{\bar{y}}, \bar{s})=(0,0, \ldots, 0, \overline{\overline{0}}, \bar{s})$ then obviously $t=0$ and $\overline{\bar{y}}=\overline{\overline{0}}$. So none of the $\Sigma^{1^{r}}$-points of $F$ are double points of $F$ which is contradiction.

If $f: M^{n} \rightarrow N^{n+k}$ is actually a prim map with lifting $g: M^{n} \rightarrow N^{n+k} \times \mathbb{R}$ and $x \in \Sigma^{1_{r}}(f) \backslash \Sigma^{1_{r+1}}(f)$, then we can take the Euclidean coordinates around $x$ and $f(x)$ introduced at the beginning of this section, and choose a last extra coordinate around $g(x)$ such that $g$ takes the local form $G(x)=(F(x), t)$. Let $j<r$ and let us consider the set

$$
A^{\prime}=\left\{(u, v) \in \mathbb{R}^{n} \times \mathbb{R}^{n}: u \in \Sigma^{1_{j}}(F), F(u)=F(v), t(u) \geq t(v)\right\}
$$

and its closure $A=\operatorname{cl}\left(A^{\prime}\right)$.

Theorem 4.3 The set $A$ is a manifold with boundary $\partial A=\left\{(u, u): u \in \Sigma^{1_{j+1}}(F)\right\}$.

Proof Theorem 4.2 implies that a boundary point of $A^{\prime}$ must be in $\Sigma^{1_{j+1}}(F)$. We shall give an explicit smooth parametrization of $A^{\prime}$ on an open halfspace, and show that this extends smoothly and bijectively to a parametrization of $\Sigma^{1_{j+1}}(F)$ on the boundary of the halfspace. It is obvious that the variables $\bar{s}$ play no role whatsoever, so without loss of generality we may assume that $z=0$ and omit $\bar{s}$ from the further calculations.

The condition $F(u)=F(v)$ obviously implies $\overline{\bar{y}}(u)=\overline{\bar{y}}(v)$, so $(u, v) \in A^{\prime}$ if and only if $t(u)>t(v)$, and $p_{i}^{\prime}(t(u))=p_{i}^{\prime \prime}(t(u))=\cdots=p_{i}^{(j)}(t(u))=p_{i}(t(u))-p_{i}(t(v))=0$ for every $i$. (Here we think of $p_{i}$ as a polynomial of one variable. Its coefficients depend on $\overline{\bar{y}}$, but since $\overline{\bar{y}}$ is independent of $u$ and $v$, this notation makes sense.)

We claim that for any choice of parameters $t(v)>t(u), \bar{y}_{r}, \bar{y}_{r-1}, \ldots, \bar{y}_{j+2}$ there is a unique choice of $\bar{y}_{j+1}, \ldots, \bar{y}_{1}$ depending smoothly on the parameters such that 
the resulting pair of points $(u, v) \in A^{\prime}$. (In case of $j=r-1$ there is only a single parameter $t(v)>0$.)

Let us first deal with the case $j<r-1$. Then for each $i$ the problem of finding $y_{j+1, i}, y_{j, i}, \ldots, y_{1, i}$ such that $p_{i}^{\prime}(t(u))=p_{i}^{\prime \prime}(t(u))=\cdots=p_{i}^{(j)}(t(u))=p_{i}(t(u))-$ $p_{i}(t(v))=0$ holds can be solved independently of each other. In fact the problem is the same for every $i$, so we fix an arbitrary $i$ and denote $p_{i}(t)=p(t)=\lambda_{r} t^{r}+$ $\cdots+\lambda_{1} t$ temporarily. Let us write $p(t)=q(t)+\lambda_{j+2} t^{j+2}+\cdots+\lambda_{r} t^{r}=q(t)+$ $r(t)$. Since $\lambda_{r}, \ldots, \lambda_{j+2}, t(u)$ and $t(v)$ are fixed parameters, we know the value of $r(t(u)), r(t(v)), r^{\prime}(t(u)), r^{\prime \prime}(t(u)), \ldots, r^{(j)}(t(u))$. We have to find the coefficients of $q$. Let us write $q$ as a Taylor polynomial around $t(u)$. Then

$$
q(t)=q(t(u))+\sum_{i=1}^{j} q^{(i)}(t(u)) \cdot \frac{(t-t(u))^{i}}{i !}+\lambda_{j+1} \cdot(t-t(u))^{j+1} .
$$

Since $0=p^{(i)}(t(u))=q^{(i)}(t(u))+r^{(i)}(t(u))$, in (1) the only unknown value is $\lambda_{j+1}$. By definition

$$
q(t(v))-q(t(u))=p(t(v))-r(t(v))+r(t(u))-p(t(u))=r(t(u))-r(t(v)),
$$

and hence by substituting $t=t(v)$ in (1) we get that

$$
\lambda_{j+1}=\left(\frac{1}{t(v)-t(u)}\right)^{j+1} \cdot\left(r(t(u))-r(t(v))-\sum_{i=1}^{j} q^{(i)}(t(u)) \cdot \frac{(t-t(u))^{i}}{i !}\right) .
$$

As every quantity on the right hand side is fixed and $t(u)>t(v)$ we find that the parameters uniquely and smoothly determine $\lambda_{j+1}$. Then all the remaining $\lambda$ 's are uniquely and smoothly determined by the Taylor expansion (1). Finally to see what happens on the boundary of the halfspace $t(u)>t(v)$ just observe, that the vanishing of the derivatives of $p$ at $t(u)$ imply that $p(t)=p(t(u))+(t-t(u))^{j+1} \cdot w(t)$ for some polynomial $w(t)$. Then the equation $p(t(v))=p(t(u))$ is equivalent to $w(t(v))=0$. Then if $t(v)-t(u)$ converges to 0 the solution will converge to a $w(t)$ for which $w(t(u))=0$, which is equivalent to saying that $p^{(j+1)}(t(u))=0$. So the boundary of the halfspace $t(u)>t(v)$ parametrizes those points $(u, u)$ for which $p^{\prime}(t(u))=p^{\prime \prime}(t(u))=\cdots=p^{(j+1)}(t(u))=0$ which is equivalent to $u \in \Sigma^{1_{j+1}}(F)$.

Now consider the case $j=r-1$. The only parameter is $t(v)$. Let us suppose that we have a solution $u$ that satisfies all the equations. Let $i \geq 1$. Then $p_{i}(t)$ is a degree $r$ polynomial for which the first $r$ derivatives vanish at $t(u)$. Thus $p_{i}=c_{i} \cdot(t-t(u))^{r}$. Further we know that $p_{i}(t(v))=p_{i}(t(u))=0$ while $t(v)>t(u)$. This is only possible if $c_{i}=0$. So all the $p_{i}$ 's must be identically 0 , except for $p_{0}$. Let us temporarily 
denote $p_{0}(t)=p(t)=t^{r+1}+\lambda_{r-1} t^{r-1}+\cdots+\lambda_{1} t$. The constraints on the derivatives imply that

$$
p(t)=p(t(u))+p^{(r)}(t(u)) \cdot \frac{(t-t(u))^{r}}{r !}+(t-t(u))^{r+1} .
$$

The polynomial $p$ has no $x^{r}$ term by definition, so $p^{(r)}(t(u))=r !(r+1) t(u)$, thus

$$
p(t)=p(t(u))+(t+r \cdot t(u))(t-t(u))^{r} .
$$

Finally

$$
p(t(u))=p(t(v))=p(t(u))+(t(v)+r \cdot t(u))(t(v)-t(u))^{r},
$$

so $t(u)=-t(v) / r$, and $p(0)=0$ determines $p(t(u))$. Thus indeed for any $t(v)>0$ there is a unique solution $u$, this solution is smoothly parametrized by $t(v)$, and the boundary $t(v)=0$ goes to the only $\Sigma^{1_{r}}$-point, the origin.

\section{References}

[1] V I Arnold, V V Goryunov, O V Lyashko, V A Vasil' ev, Singularity theory. I, from: "Dynamical systems. VI", Encyclopaedia Math. Sci. 6, Springer, Berlin (1993) iv+245 MR1230637 Translated from the 1988 Russian original by A Iacob

[2] C McCrory, Cobordism operations and singularities of maps, Bull. Amer. Math. Soc. 82 (1976) 281-283 MR0415637

[3] C McCrory, Geometric homology operations, from: "Studies in algebraic topology", (G-C Rota, editor), Adv. in Math. Suppl. Stud. 5, Academic Press, New York (1979) 119-141 MR527247

[4] F Ronga, On multiple points of smooth immersions, Comment. Math. Helv. 55 (1980) 521-527 MR604710

[5] A Szúcs, On the cobordism groups of immersions and embeddings, Math. Proc. Cambridge Philos. Soc. 109 (1991) 343-349 MR1085401

[6] A Szúcs, On the singularities of hyperplane projections of immersions, Bull. London Math. Soc. 32 (2000) 364-374 MR1750481

Rényi Alfréd Institute of Mathematics

Budapest, Hungary

lipi@renyi.hu

Received: 21 May 2008 Revised: 19 February 2009 